University of New Orleans

ScholarWorks@UNO

6-1-1992

\title{
Division-of-amplitude photopolarimeter based on conical diffraction from a metallic grating
}

R. M.A. Azzam

University of New Orleans, razzam@uno.edu

Follow this and additional works at: https://scholarworks.uno.edu/ee_facpubs

Part of the Electrical and Electronics Commons

\section{Recommended Citation}

R. M. A. Azzam, "Division-of-amplitude photopolarimeter based on conical diffraction from a metallic grating," Appl. Opt. 31, 3574-3576 (1992)

This Article is brought to you for free and open access by the Department of Electrical Engineering at ScholarWorks@UNO. It has been accepted for inclusion in Electrical Engineering Faculty Publications by an authorized administrator of ScholarWorks@UNO. For more information, please contact scholarworks@uno.edu. 


\section{Division-of-amplitude photopolarimeter based on conical diffraction from a metallic grating}

\section{R. M. A. Azzam}

The author is with the Department of Electrical Engineering, University of New Orleans, Lakefront, New Orleans, Louisiana 70148.

Received 10 December 1991.

Sponsored by Thomas K. Gaylord, Georgia Institute of Technology, Atlanta, Georgia. 0003-6935/92/193574-03\$05.00/0.

(c) 1992 Optical Society of America.

A new photopolarimeter that employs polarization-dependent conical diffraction of light by a metallic grating for the simultaneous measurement of the four Stokes parameters of light is described.

In a division-of-amplitude photopolarimeter ${ }^{1,2}$ (DOAP) an incident light beam, whose four Stokes parameters are to be measured, is split into four beams by using an appropriately coated $^{2}$ beam splitter and two Wollaston prisms or equivalent birefringent or multilayer-coated polarizing beam splitters (Fig. 1). Linear detection of the light fluxes of the four component beams produces four output electrical signals that determine the four Stokes parameters by means of an instrument matrix that is obtained by calibration. The DOAP is capable of a fast (time-resolved) measurement of the most general state of partial elliptical polarization of light since it employs no moving parts or modulators. A variant of $\mathrm{DOAP}^{3}$ and three instruments that are based on the original description ${ }^{1}$ have been operated successfully. ${ }^{4-6}$

The fact that a diffraction grating produces multiple beams from an incident beam and the significant polarization effects that are known to accompany grating diffraction $^{7-9}$ make the grating an ideally suited element for DOAP. Here we describe such a grating-based DOAP (G-DOAP) and report briefly on the successful testing of a simple version of this instrument, in which the grating alone replaces all three beam splitters of the original DOAP. ${ }^{1}$ Figure 2 is a schematic diagram of a G-DOAP. A metallic reflection-diffraction grating $\mathrm{G}$ breaks the incident light beam of Stokes vector $\mathbf{S}=\left[S_{0} S_{1} S_{2} S_{3}\right]^{t}$, where $t$ indicates the transpose, into at least four diffracted orders. To make the diffraction efficiency of each diffracted order sensitive to $S_{2}$ and $S_{3}$ the grating is set for oblique incidence at an angle $\phi$ and the grating lines are inclined at an arbitrary angle $\alpha$ with respect to the plane of incidence. In the conventional (spectrometer) orientation of the grating, in which the lines are normal to the plane of incidence (i.e., $\alpha=90^{\circ}$ ), the diffraction efficiency becomes independent of $S_{2}$ and $S_{3}$ and the G-DOAP cannot function as a complete polarimeter. The $m$ th diffracted beam is intercepted by a linear photodetector $\mathrm{D}_{m}$ and generates an output electrical signal $i_{m}(m=0,1,2, \ldots, M-1)$ that is proportional to the power carried by that order. (The diffracted orders and signals are numbered in the manner described in Fig. 2.) Consequently the output signal of the $m$ th detector is a linear combination of the four Stokes parameters of the incident light, i.e.,

$$
i_{m}=\sum_{n=0}^{3} a_{m n} S_{n}, \quad m=0,1,2, \ldots, M-1 .
$$

The $m$ th projection vector $\left(a_{m 0} a_{m 1} a_{m 2} a_{m 3}\right)$ is determined by the first row of the Mueller matrix of diffraction of the $m$ th order multiplied by a scale factor that represents the sensitivity of the photodetector $\mathrm{D}_{m}$.

When only four orders are detected, the corresponding set of four signals $i_{m}, m=0,1,2$, and 3 , defines a current vector $\mathbf{I}=\left[i_{0} i_{1} i_{2} i_{3}\right]^{t}$ that is linearly related to the input Stokes vector $\mathbf{S}$ by

$$
\mathbf{I}=\mathscr{N} \mathbf{S} \text {. }
$$

The $4 \times 4$ instrument matrix $\mathscr{A}=\left(a_{m n}\right)$ is characteristic of the G-DOAP at a given wavelength. For a given grating $\mathscr{A}$ is a function of the selected diffracted orders (if more than four are available), the angular orientation parameters $\phi$ and $\alpha$, and the wavelength of light $\lambda$. When $\alpha \neq 0$, i.e., for an arbitrary orientation that produces conical diffraction, ${ }^{9} \mathscr{A}$ is expected to be nonsingular generally. From Eq. (2) $\mathbf{S}$ is then given by

$$
\mathbf{S}=\mathscr{M}^{-1} \mathbf{I}
$$

The instrument matrix $\mathscr{A}$ of the G-DOAP is determined by calibration by the same procedures established for the four-detector photopolarimeter..$^{10,11}$

The validity of the principle of the G-DOAP is established experimentally by using an Al-coated 600 -groove $/ \mathrm{mm}$ holographic (spectrogon) grating set at a $65^{\circ}$ angle of incidence with the grooves inclined at $45^{\circ}$ with respect to the plane of incidence. A He-Ne laser with a 633-nm wavelength is used as the source and small-area p-i-n photodiodes with integral adjustable-gain operational amplifiers are used as detectors. The grating diffracts three orders (out of the plane of incidence) plus the specular zero-order beam for a total of four. Calibration is carried out by using the method of Ref. 11 and the resulting instrument matrix is

$$
\mathscr{A}=\left[\begin{array}{rrrr}
1.35 & -0.60 & 0.57 & 0.39 \\
1.57 & -0.05 & 0.28 & 0.06 \\
2.36 & 0.55 & -0.62 & -0.25 \\
0.92 & -0.17 & 0.14 & 0.01
\end{array}\right] .
$$

The four diffracted orders are of comparable powers and the gain settings of all postdetection amplifiers are identical. The precision with which the elements 


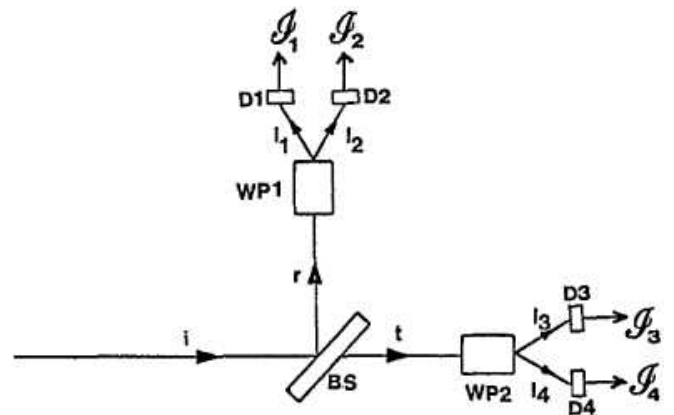

Fig 1. DOAP with three beam splitters BS, WP1, and WP2 (after Ref. 1).

are determined with a plurality of calibration states ${ }^{11}$ is of the order of \pm 0.01 . This can be improved by chopping the incident beam and using lock-in detection (which we have not done so far). The determinant of the matrix of Eq. (4) equals 0.068, and the matrix is not nearly singular. ${ }^{12}$ For comparison the determinant of $\mathscr{A}$ in the singular orientation $\alpha=90^{\circ}$ is found experimentally to be 3 orders of magnitude lower. In its preliminary unoptimized version the G-DOAP measures the normalized Stokes parameters with a precision of \pm 0.01 , which is comparable with that of the first four-detector polarimeter. ${ }^{10}$

A measure of the overall polarization sensitivity of the individual diffracted orders is given by the lengths of the normalized projection vectors. ${ }^{13}$ From Eq. (4) one calculates

$$
\begin{array}{ll}
\left|\mathbf{a}_{0}\right|=0.682, & \left|\mathbf{a}_{1}\right|=0.182, \\
\left|\mathbf{a}_{2}\right|=0.365, & \left|\mathbf{a}_{3}\right|=0.239 .
\end{array}
$$

Thus the zero and second orders are more polarization sensitive than the first and third orders. This also extends to the sensitivity to the right- and left-handed circular polarization states as represented by the elements of the last column of $\mathscr{A}$.

The G-DOAP offers a considerable range of advantage and flexibility:

(1) In common with the DOAP and the fourdetector photopolarimeter, the G-DOAP has no mov-

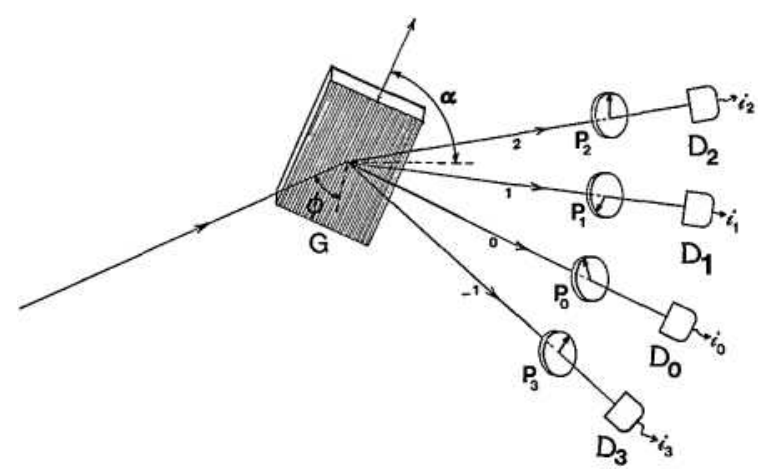

Fig 2. G-DOAP in which a diffraction grating $\mathrm{G}$ performs the function of the three beam splitters of the DOAP in Fig. 1. $\mathrm{P}_{m}$ $(m=0,1,2, \ldots)$ are optional linear polarizers. ing parts or modulators; hence it is capable of timeresolved polarization measurements with a speed that is limited by the photodetectors and associated electronics only.

(2) The polarization sensitivity of the G-DOAP can be substantially improved by the insertion of linear (e.g., dichroic sheet) polarizers $\left(\mathrm{P}_{0}, \mathrm{P}_{1}, \mathrm{P}_{2}\right.$, and $\mathrm{P}_{3}$ in Fig. 2) in front of the respective detectors. This, in effect, lengthens each normalized projection vector to nearly the maximum value of 1 and increases the determinant of the instrument matrix. The orientations of the polarizers can be chosen to optimize performance at a given wavelength.

(3) Optimum conditions can also be sought by controlling the groove shape, the orientation angles $\phi$ and $\alpha$, and by applying thin-film dielectric coatings on the grating surface.

(4) By choosing a grating with a lower spatial frequency (e.g., $100-300 \mathrm{~g} / \mathrm{mm}$ for visible light) a number of beams $M>4$ (distributed around a cone with apex at the point of reflection) becomes available. When each beam is intercepted with a photodetector, $M$ output signals that represent $M$ projections of the Stokes vector $\mathbf{S}$ are obtained. With these additional (redundant) projections a more accurate and precise estimate of $\mathbf{S}$ can be determined. In this case, Eq. (2) still holds except that $I$ is now an $M \times 1$ vector and $\mathscr{A}$ is an $M \times 4$ rectangular matrix $(M>4)$. A least-squares solution for $S$ is given by ${ }^{14}$

$$
\mathbf{S}=\left(\mathscr{A}^{t} \mathscr{A}\right)^{-1} \mathscr{A} \mathbf{I} \text {. }
$$

(5) Perhaps the most important advantage of the G-DOAP is that it can be adapted to perform timeresolved spectropolarimetry. This can be achieved by replacing each detector with a detector array. The instrument, however, requires calibration over the wavelength range of interest with a suitable polarization state generator (which need not be perfect). ${ }^{11}$

In summary, a novel instrument that uses multiplebeam conical diffraction from a metal grating to perform time-resolved and spectroscopic photopolarimetry has been described. This also provides yet another interesting and potentially important application of grating diffraction. ${ }^{15}$

I thank Kurt Giardina for assembling and testing the G-DOAP.

\section{References}

1. R. M. A. Azzam, "Division-of-amplitude photopolarimeter (DOAP) for the simultaneous measurement of all four Stokes parameters of light," Opt. Acta 29, 685-689 (1982).

2. R. M. A. Azzam, "Beam splitters for the division-of-amplitude photopolarimeter (DOAP)," Opt. Acta 32, 767-777 (1985).

3. G. E. Jellison, "Four-channel polarimeter for time-resolved ellipsometry," Opt. Lett. 12, 766-769 (1987).

4. K. Brudzewski, "Static Stokes ellipsometer: general analysis and optimization," J. Mod. Opt. 38, 889-896 (1991).

5. S. Krishnan, Intersonics, Inc., Northbrook, Ill. (personal communication). 
6. J. Morel, Université de Neuchâtel, Neuchâtel, Switzerland (personal communication).

7. C. F. Meyer, The Diffraction of Light, X-Rays, and Material Particles (Edwards, Ann Arbor, Mich., 1949), pp. 136-137.

8. J. M. Bennett and H. E. Bennett, "Polarization," in Handbook of Optics, W. G. Driscoll and W. Vaughan, eds. (McGraw-Hill, New York, 1978), pp. 10-76-10-77.

9. M. G. Moharam and T. K. Gaylord, "Three-dimensional vector coupled-wave analysis of planar grating diffraction," J. Opt. Soc. Am. 73, 1105-1112 (1983)

10. R. M. A. Azzam, E. Masetti, I. M. Elminyawi, and F. G. Grosz, "Construction, calibration, and testing of a four-detector photopolarimeter,' Rev. Sci. Instrum. 59, 84-88 (1988).

11. R. M. A. Azzam and A. G. Lopez, "Accurate calibration of the four-detector photopolarimeter with imperfect polarizing optical elements," J. Opt. Soc. Am. A 6, 1513-1521 (1989).

12. B. Noble, Applied Linear Algebra (Prentice-Hall, Englewood Cliffs, N.J., 1969), p. 242.

13. R. M. A. Azzam, "Instrument matrix of the four-detector photopolarimeter: physical meaning of its rows and columns and constraints on its elements," J. Opt. Soc. Am. A 7, 87-91 (1990).

14. B. Noble, Applied Linear Algebra (Prentice-Hall, Englewood Cliffs, N.J., 1969), p. 142.

15. T. K. Gaylord and M. G. Moharam, "Analysis and applications of optical diffraction by gratings," Proc. IEEE 73, 894-937 (1985). 